\title{
A 6-Gb/s Differential Voltage Mode Driver with Independent Control of Output Impedance and Pre- Emphasis Level
}

\author{
Chang-Hyun Bae, Dong-Ho Choi, Keun-Seon Ahn, and Changsik Yoo
}

\begin{abstract}
A 6-Gb/s differential voltage mode driver is presented whose output impedance and pre-emphasis level can be controlled independently. The voltage mode driver consists of five binary-weighted slices each of which has four sub-drivers. The output impedance is controlled by the number of enabled slices while the pre-emphasis level is determined by how many sub-drivers in the enabled slices are driven by post-cursor input. A prototype transmitter with a voltage-mode driver implemented in a $65-\mathrm{nm}$ CMOS logic process consumes $34.8-\mathrm{mW}$ from a $1.2-\mathrm{V}$ power supply and its pre-emphasized output signal shows 165-mVpp,diff and 0.56-UI eye opening at the end of a cable with 10-dB loss at 3-GHz.
\end{abstract}

Index Terms-Voltage-Mode driver, impedance calibration, pre-emphasis, CMOS

\section{INTRODUCTION}

A current-mode differential driver has been more popular than a voltage-mode differential driver in a highspeed serial link for its immunity to power supply noise

Manuscript received Nov. 9, 2012; accepted May. 28, 2013 A part of this work was supported by Seoul R\&BD Program (SS100022) and IT R\&D Program (Large Scale Hyper-MLC SSD Technology Development, No. 10035202) of MKE/KEIT, Korea. The CAD tools were provided by IDEC, Korea.

C.-H. Bae is with the Integrated Circuits Lab. Hanyang University, Seoul 133-791, Korea and also with the DRAM Design Department of Samsung Electronics, Hwasung, Korea. D.-H. Choi, K.-S. Ahn, and C. Yoo are with the Integrated Circuits Lab. Hanyang University, Seoul 133791, Korea

E-mail : csyoo@hanyang.ac.kr and ease of impedance matching [1]. For the same voltage swing, the power consumption of a current-mode driver, however, is larger than that of a voltage-mode driver and therefore a voltage-mode driver is drawing lots of interest especially for low-power serial link [2, 3].

To ensure signal integrity, the output impedance of a voltage-mode driver has to be matched to the characteristic impedance of signal channel which is normally $50-\Omega[4]$. For output impedance control, a voltage mode driver is usually implemented with multiple slices which are enabled selectively to get the desired output impedance $[5,6]$. As the data rate increases, the inter-symbol interference (ISI) becomes much more sever and the signal equalization of a receiver may not be sufficient to get enough eye opening and the pre-emphasis of transmitted signal level is often required. The pre-emphasis is usually achieved by employing a sub-driver which may increase or decrease the transmitted signal level depending on the pattern $[7,8]$. The required level of pre-emphasis can be different depending on the channel loss and thus the sub-driver of a voltage-mode driver must have variable strength. The variable strength of the sub-driver, however, makes the calibration of the output impedance very complicated because it can also change the output impedance.

A couple of techniques are presented which allow the independent control of the output impedance and preemphasis level. In [9], a voltage-mode driver is implemented with 15 slices and the output impedance is controlled by adjusting the driving gate voltage with an on-chip linear voltage regulator and the pre-emphasis level is controlled by changing the number of slices used 
for the main- and sub-driver. In [10], the multiple slices of a voltage-mode driver are further divided into binaryweighted sub-units. The number of slices is calibrated for the output impedance control and the pre-emphasis level is controlled by changing the number of subunits allocated for the main tap and post-cursor tap. Although a complex analog linear voltage regulator is not required, this scheme is still very complex because 22 identical slices with five binary weighted sub-units are required. For the control of the output impedance and preemphasis level, the pull-up (pull-down) path of each subunit has two pMOS (nMOS) transistors and a passive resistor in series, which may increase the size of the transistors and the parasitic capacitance in turn to keep the ratio of the resistance of the transistors to that of the passive resistor.

In this paper, a $6-\mathrm{Gb} / \mathrm{s}$ differential voltage-mode driver with an independent control of output impedance and pre-emphasis level is presented. The output impedance and pre-emphasis level are all controlled at the pre-driver to simplify the design of the voltage-mode driver. The output impedance is calibrated with an external reference resistor during the wafer test by a successive approximation register (SAR) based control logic. While the voltage-mode driver operates with a $1.2-\mathrm{V}$ supply, the voltage swing is adjusted by a linear regulator which provides a $0.3-\mathrm{V}$ supply.

Section II and III explains the differential voltagemode driver whose experimental results are given in Section IV. Finally, the paper is concluded in Section V

\section{ARCHITECTURE}

Fig. 1 shows the architecture of the 6-Gbps transmitter where the differential voltage-mode driver is employed. The 300-Mbps parallel data input $D_{I N}<19: 0>$ is serialized to the 6-Gbps data $D Q<n>$ while $D Q<n-1>$ is used for the pre-emphasis. The differential voltage-mode driver consists of five binary-weighted slices each of which has four sub-drivers for both the positive and negative outputs. Each sub-driver requires separate driving signals for pull-up and pull-down. For the positive (negative) output of the differential voltage-mode driver, the predriver generates $P D R V_{P}<19: 0>\left(N D R V_{P}<19: 0>\right)$ and $P D R V_{N}<19: 0>\left(N D R V_{N}<19: 0>\right)$ which drive the pull-up and pull-down transistors, respectively.

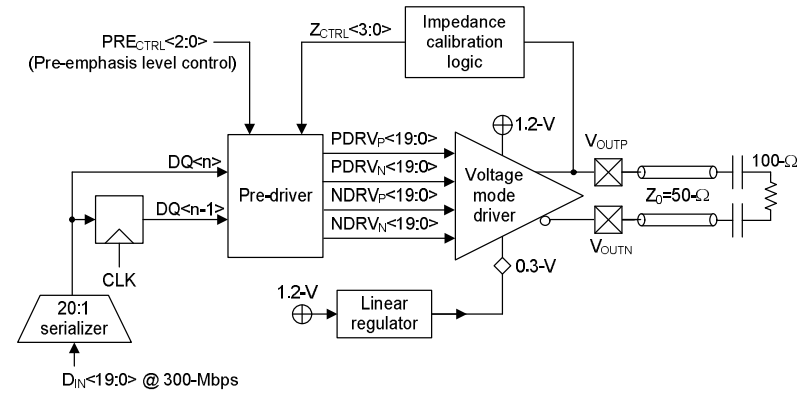

Fig. 1. 6-Gbps transmitter with a voltage-mode driver.

Because the output voltage swing of a voltage-mode driver is determined by its supply voltage, the voltagemode driver in [10] uses on-chip generated high- and low-supply voltages. Because the voltage-mode driver of this work uses a $65-\mathrm{nm}$ CMOS technology, the maximum allowed high-supply voltage level is $1.2-\mathrm{V}$. If an on-chip generated high-supply voltage lower than $1.2-\mathrm{V}$ is used to control the voltage swing, the size of pMOS transistors of the voltage-mode driver would have to be increased because the over-drive voltage becomes smaller than 1.2$\mathrm{V}$. In this work, the high-supply voltage is fixed to $1.2-\mathrm{V}$ in order not to increase the size of the pull-up pMOS transistors and only the low-supply voltage of $0.3-\mathrm{V}$ is generated on-chip to control the voltage swing as shown in Fig. 1 and the link is AC-coupled.

The output impedance and pre-emphasis level are controlled at the pre-driver by the control codes $Z_{C T R L}<3: 0>$ and $P R E_{C T R L}<2: 0>$, respectively. The control code $Z_{\text {CTRL }}<3: 0>$ of the output impedance is generated during the wafer test by the impedance calibration logic. The output impedance and pre-emphasis level can be adjusted independently with each other, which allows simple control.

\section{CIRCUIT DESCRIPTION}

\section{Pre-driver}

The pre-driver shown in Fig. 2 generates $P D R V_{P}<19: 0>$ and $P D R V_{N}<19: 0>$ for the pull-up and pull-down of the positive output, respectively. The pulldown strength of the pre-driver sub-cells generating $P D R V_{P}<k>$ and pull-up strength of the pre-driver subcells generating $P D R V_{N}<k>$ are intentionally weakened 


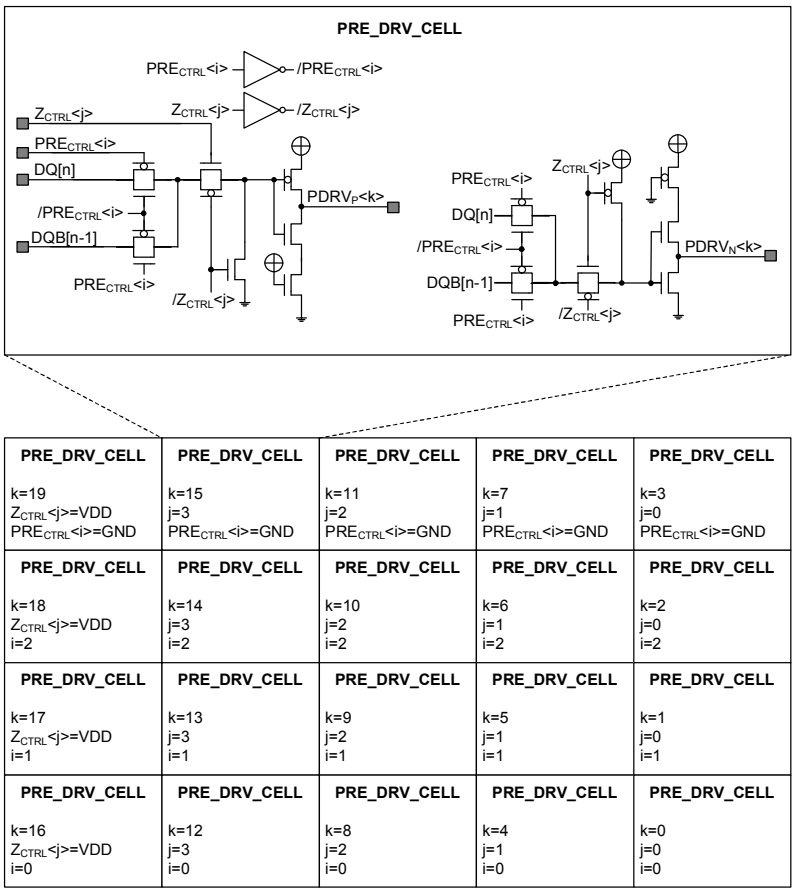

Fig. 2. Pre-driver generating the driving signals $P D R V_{P}<19: 0>$ and $P D R V_{N}<19: 0>$ of the pMOS and nMOS transistors, respectively of the voltage-mode driver for the positive output $V_{\text {OUTP. }}$. The driving signals $N D R V_{P}<19: 0>$ and $N D R V_{N}<19: 0>$ of the voltage-mode driver for the negative output $V_{\text {OUTN }}$ are generated by the same type of pre-driver whose inputs are $D Q B<n>$ and $D Q<n-1>$. The pre-emphasis level and output impedance of the voltage-mode driver are all controlled at the pre-driver independently by $P R E_{C T R L}<2: 0>$ and $Z_{C T R L}<3: 0>$.

in order to prevent the through-current in the voltagemode driver [11]. If $Z_{C T R L}<j>=$ " 0 ", the outputs $P D R V_{P}<k>$ and $P D R V_{N}<k>$ of the corresponding subcells of the pre-driver are " 1 " and " 0 ", respectively, which turns off the sub-drivers of the voltage-mode driver driven by them.

For the pre-emphasis level control, $P R E_{C T R L}<i>$ is selectively set to either "1" or " 0 ". If $P R E_{C T R L}<i>=$ "1", the corresponding pre-driver sub-cells accept $D Q B<n-1>$ as the input, which increases the pre-emphasis level. For the negative output of the differential voltage-mode driver, $N D R V_{P}<19: 0>$ and $N D R V_{N}<19: 0>$ for pull-up and pull-down are generated by the same type of pre-driver whose input is $D Q B<n>$ and $D Q<n-1>$.

\section{Differential voltage-mode driver}

The differential voltage-mode driver shown in Fig. 3 consists of two single-ended voltage-mode drivers which consist of 20 sub-drivers. Because the output impedance and pre-emphasis level are all controlled at the pre-driver, the differential voltage-mode driver is very simple. Each sub-driver has one pull-up pMOS transistor, one pulldown nMOS transistor, and a passive resistor. The output resistance of each sub-driver shown in the figure is the sum of the resistances of the pull-up or pull-down transistor and passive resistor. The voltage-mode driver operates with the $1.2-\mathrm{V}$ high- and $0.3-\mathrm{V}$ low-supply voltages.

The single-ended voltage-mode driver has five slices which have four weighted sub-drivers. Among the five slices, SLICE $<4>$ is always enabled and $S L I C E<j>$ is selectively enabled by the output impedance control code $Z_{\text {CTRL }}<j>$ where $j=3 \sim 0$. The output resistances of the slices are binary weighted as shown in the figure and therefore the output impedance of the voltage-mode driver can be controlled in 16 steps. Among the four weighted sub-drivers of each slice, the upper-most ones are always used for the main tap $D Q<n>$ while the other three sub-drivers are used for either the main tap $D Q<n>$ or the post-cursor tap $D Q B<n-1>$ according to the control code $P R E_{C T R L}<2: 0>$. Therefore, the pre-emphasis level can be controlled in eight steps. The output resistance of each sub-driver does not change regardless whether it is used for the main or post-cursor tap.

Because the output impedance is controlled by selectively enabling the slices while the pre-emphasis level is controlled at the sub-driver level of each slice, the two parameters can be independently calibrated.

\section{Impedance Calibration}

The output impedance of the differential voltage-mode driver has to be matched to the characteristic impedance $50-\Omega$ of the transmission channel. The sizes of the pullup and pull-down transistors are determined so their resistances are $1 / 10$ of the resistance of the passive resistor. Therefore, the variation of the output impedance due to the temperature and supply voltage changes is not large and only the process variation has to be calibrated.

In order to calibrate the output impedance over the process variation, the automatic impedance calibration circuit is enabled during the wafer test as shown in Fig. 4(a). An external reference resistor $R_{R E F}=100-\Omega$ is connected between the positive and negative outputs 


\begin{tabular}{|c|c|c|c|c|}
\hline 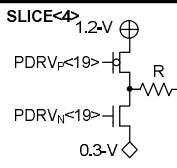 & $\begin{array}{c}\text { SLICE }<3>_{1.2-V} \oplus \\
P D R V_{P}<15>-15 \\
P D R V_{N}<15>-15 \\
0.3-V \diamond\end{array}$ & 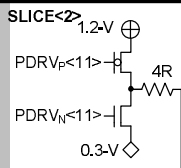 & 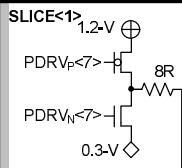 & $\begin{array}{c}\text { SLICE }<0>1.2-V \varphi \\
P D R V_{P}<3>-b Q \\
P D R V_{N}<3>-15 \\
0.3-V \diamond\end{array}$ \\
\hline 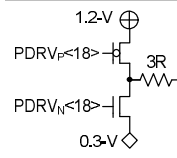 & 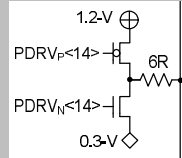 & 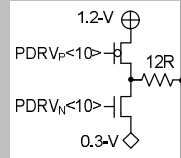 & 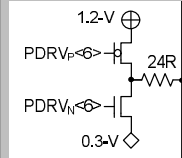 & 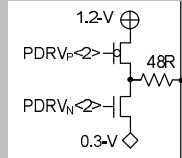 \\
\hline 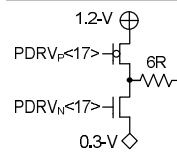 & 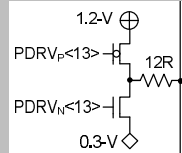 & 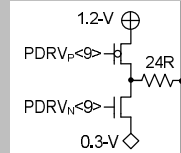 & $\mathrm{PDRV}_{\mathrm{P}}<5>-\mathrm{V} \mathrm{N}_{\mathrm{N}}<5>-1 . \mathrm{V}$ & 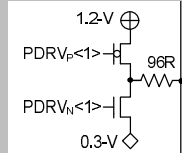 \\
\hline 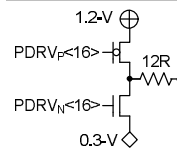 & $\begin{array}{c}1.2-V \Phi \\
P D R V_{P}<12>-T C \\
P D R V_{N}<12>-15 \\
0.3-V \diamond\end{array}$ & 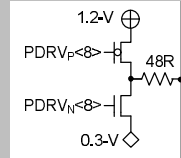 & 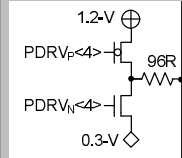 & 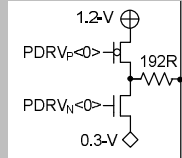 \\
\hline 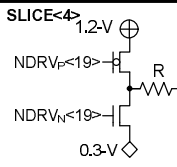 & 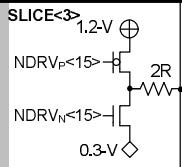 & 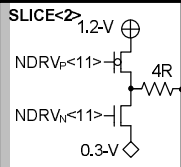 & 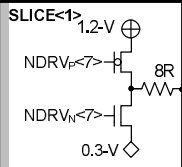 & $\begin{array}{c}\text { SLICE }<0>1.2-V \varphi \\
N D R V_{P}<3>-10 \\
N D R V_{N}<3>-15 \\
0.3-V \diamond\end{array}$ \\
\hline 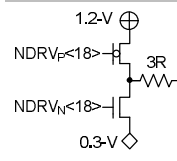 & 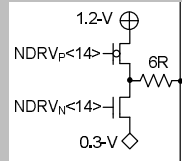 & 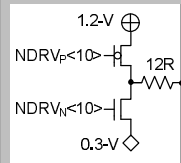 & 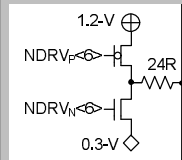 & 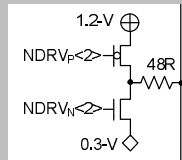 \\
\hline 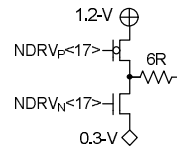 & 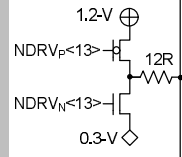 & 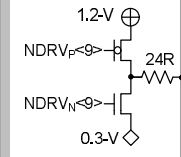 & 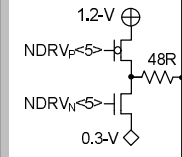 & 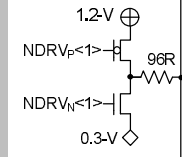 \\
\hline 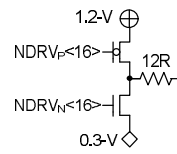 & 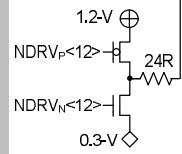 & 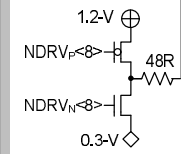 & $\begin{array}{l}\mathrm{NDR}_{N}<4>-1 \\
0.3-\mathrm{V} \diamond\end{array}$ & 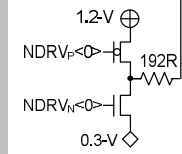 \\
\hline
\end{tabular}

Fig. 3. Voltage-mode driver. The resistance of each sub-driver is the sum of the resistances of the transistor and passive resistor.

$V_{\text {OUTP }}$ and $V_{\text {OUTN }}$. During the output impedance calibration, $D Q<n>$ is " 1 " and $D Q<n-1>$ is " 0 " in order for the voltage levels of the positive and negative outputs not to change by the pre-emphasis level control code $P R E_{\text {CTRL }}<2: 0>$. If the output impedance is denoted as $R_{\text {OUT }}$, the differential voltage-mode driver with $R_{R E F}$ forms a resistive voltage-divider as shown in Fig. 4(b). If $R_{\text {OUT }}=50-\Omega$, the positive output $V_{\text {OUTP }}$ is $0.975-\mathrm{V}$. Thus the SAR-based control logic calibrates the output impedance by forcing the voltage level of $V_{\text {OUTP }}$ to be equal to the reference voltage level of $0.975-\mathrm{V}$.

\section{EXPERIMENTAL RESULTS}

The differential voltage-mode driver was implemented in a 65-nm CMOS technology. The microphotograph of the whole chip and the detailed layout of the voltagemode driver are shown in Fig. 5. For the measurement, the chip has been mounted on a printed circuit board (PCB) and bonded. Because we do not have wafer test equipment, the impedance calibration of the voltagemode driver has been performed with a reference resistor on the PCB which can be disconnected during the normal operation.

The output impedance of the voltage-mode driver is 


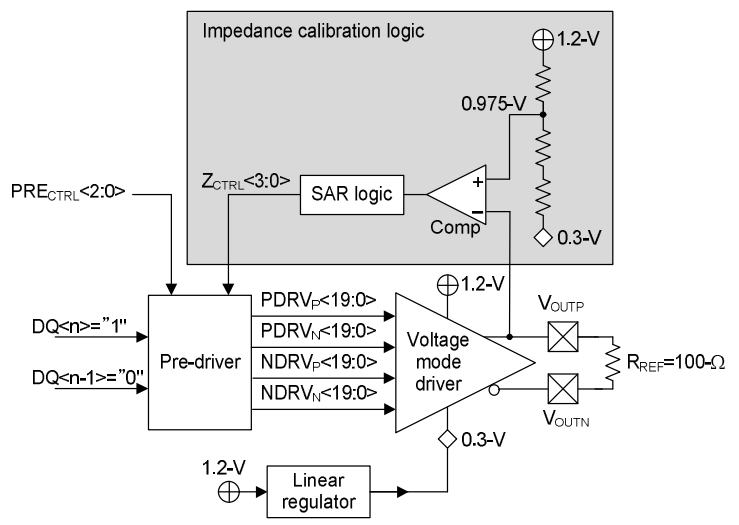

(a)

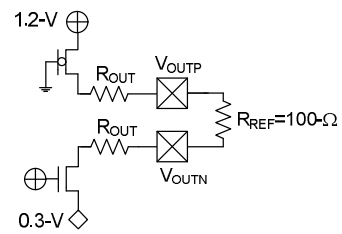

(b)

Fig. 4. (a) Configuration of the voltage-mode driver during the output impedance calibration. The reference resistor $R_{R E F}$ is an external one located on a test set-up, (b) Equivalent circuit of the differential voltage-mode driver during the impedance calibration.

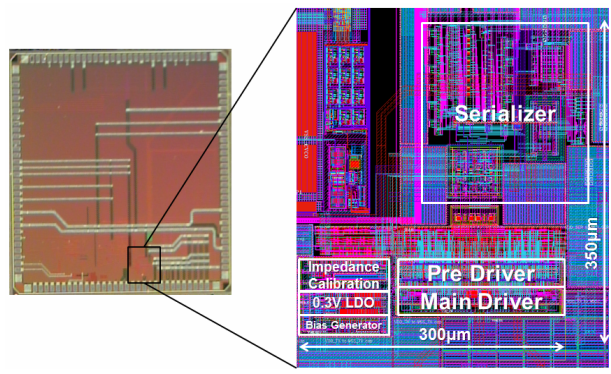

Fig. 5. Microphotograph and layout.

measured versus the impedance control code $Z_{C T R L}<3: 0>$ as shown in Fig. 6. The desired output impedance $50-\Omega$ can be obtained when $Z_{C T R L}<3: 0>=$ "0111". For a 3-Gbps input data stream of "101110", the output waveform of the voltage mode driver is overlaid for eight preemphasis levels in Fig. 7. The pre-emphasis level can be controlled from $0-\mathrm{V}$ to $165-\mathrm{mV}$ in eight steps with the step size of approximately $23.6-\mathrm{mV}$. Fig. 8 shows the eye diagram and bit-error rate (BER) bathtub curve at the end of a cable with $10-\mathrm{dB}$ loss at $3-\mathrm{GHz}$ for a $6-\mathrm{Gbps}$ pseudo-random binary sequence (PRBS) input. The horizontal and vertical openings are improved from 50-ps

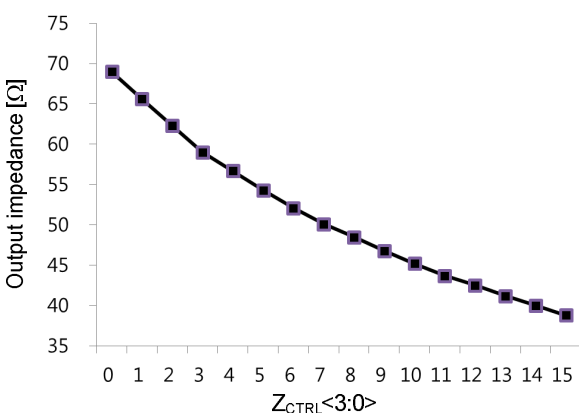

Fig. 6. Measured output impedance versus the impedance control code $Z_{C T R L}<3: 0>$

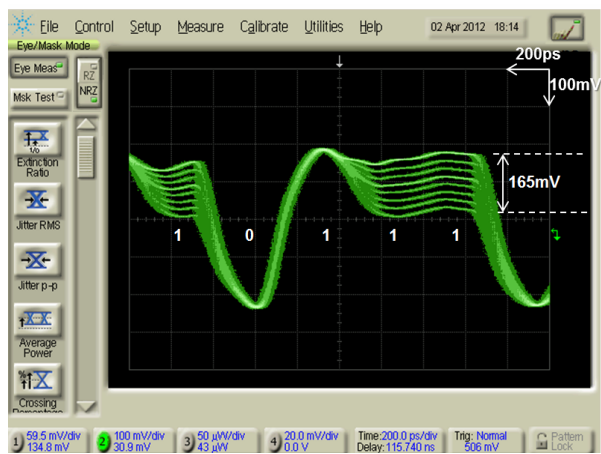

Fig. 7. Overlap of the output waveform for the eight preemphasis levels.
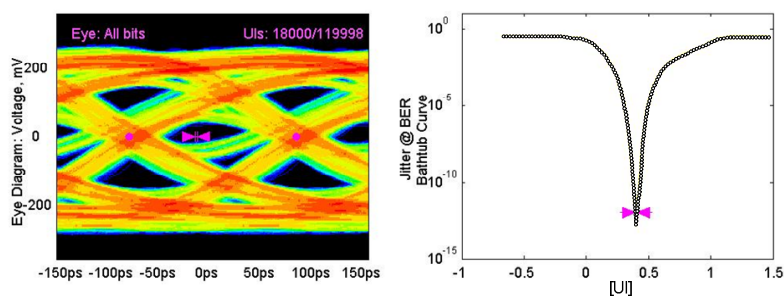

(a)
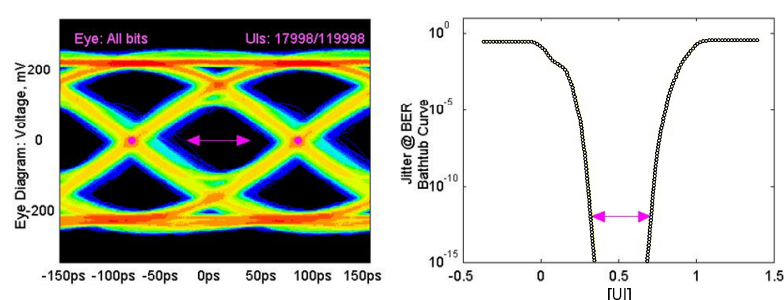

(b)

Fig. 8. Eye diagram and bit-error rate (BER) bathtub curve at the end of a cable with 10-dB loss at 3-GHz (a) without and (b) with pre-emphasis.

to 94-ps and from 42-mVpp,diff. to $165-\mathrm{mVpp}$,diff., respectively by the pre-emphasis of the differential voltage-mode driver. 
Table 1. Power consumption

\begin{tabular}{|c|c|}
\hline Block & Power \\
\hline Differential voltage-mode driver & $6-\mathrm{mW}$ \\
\hline Pre-driver & $12-\mathrm{mW}$ \\
\hline Serializer & $10.8-\mathrm{mW}$ \\
\hline Regulator \& Bias circuit & $1.2-\mathrm{mW}$ \\
\hline Clock buffer \& Repeaters & $4.8-\mathrm{mW}$ \\
\hline Total power & $\mathbf{3 4 . 8}-\mathbf{m W}$ \\
\hline
\end{tabular}

Table 2. Performance summary \& comparison

\begin{tabular}{|c|c|c|c|c|c|}
\hline & Technology & $\begin{array}{c}\text { Max. data rate } \\
{[\mathrm{Gb} / \mathrm{s}]}\end{array}$ & $\begin{array}{c}\text { Efficiency } \\
{[\mathrm{mW} / \mathrm{Gb} / \mathrm{s}]}\end{array}$ & $\begin{array}{c}\text { Impedance \& Pre-emphasis } \\
\text { level control }\end{array}$ & $\begin{array}{c}\text { Number of sub-drivers / Structure of sub- } \\
\text { driver }\end{array}$ \\
\hline$[6]$ & $65 \mathrm{~nm}$ SOI CMOS & 16 & 3.6 & dependent & 44 sub-drivers / stacked FETs \\
\hline$[10]$ & $65 \mathrm{~nm} \mathrm{CMOS}$ & 8.5 & 11.3 & independent & 110 sub-drivers / stacked FETs \\
\hline$[12]$ & $65 \mathrm{~nm} \mathrm{CMOS}$ & 20 & 8.3 & independent & 64 sub-drivers / stacked FETs \\
\hline This work & $\mathbf{6 5} \mathbf{n m ~ C M O S ~}$ & $\mathbf{6}$ & $\mathbf{5 . 8}$ & independent & $\mathbf{4 0}$ sub-drivers / non-stacked FETs \\
\hline
\end{tabular}

The power consumption of the transmitter with the voltage-mode driver has been measured with the clock pattern output and the result is summarized in Table 1. The 1.5-GHz four-phase output clocks of an internal phase-locked loop (PLL) are buffered by the clock buffers and repeaters.

The performance of the transmitter with the voltagemode driver is summarized and compared with other works in Table 2. While providing the independent control of the output impedance and pre-emphasis level, the proposed voltage-mode driver has only one transistor between the power supply rails and passive resistor, which aids to reduce the size of the transistors and in turn to decrease the variation of the output impedance of the driver due to the change of the temperature and supply level.

\section{CONCLUSIONS}

A $6-\mathrm{Gb} / \mathrm{s}$ differential voltage-mode driver in a 65-nm CMOS technology is presented. The voltage-mode driver features the independent control of output impedance and pre-emphasis level. The output impedance of the voltage mode driver is automatically calibrated by a SAR-based logic during the wafer test. The transmitter with the voltage mode driver consumes $34.8-\mathrm{mW}$ from a $1.2-\mathrm{V}$ power supply and its pre-emphasized output signal shows $165-\mathrm{mVpp}$, diff and 0.56-UI eye opening at the end of a cable with $10-\mathrm{dB}$ loss at $3-\mathrm{GHz}$.

\section{REFERENCES}

[1] M. Green and U. Singh, "Design of CMOS CML circuits for high-speed broadband communications," Proc. IEEE Int. Symp. Circuits and Systems, pp. 204-207, May, 2003.

[2] D. Heidar, M. Dessouky, H. F. Ragaie, "Comparison of output drivers for high-speed serial links," Microelectronics, pp. 329-337, ICM 2007.

[3] D. A. Yokoyama-Martin, K. Krishna, J. Stonick, A. Caffee, E. Kolet Gamble, C. Jones, J. McNeal, J. Parker, R. Segelken, J. Sonntag, K. Umino, J. Upton, D. Weinlader, and S. Wolfer, "A multistandard low power $1.5-3.125 \mathrm{~Gb} / \mathrm{s}$ serial transceiver in 90nm CMOS," Proc. IEEE Custom Integrated Circuits Conf. pp. 401-404, 2006.

[4] J. Liu and X. Lin, "Equalization in high speed communication systems," IEEE Circuits and Systems Magazine, pp. 4-15, Second Quarter 2004.

[5] K. Krishna, D. A. Yokoyama-Martin, A. Caffee, C. Jones, M. Loikkanen, J. Parker, R. Segelken, J. L. Sonntag, J. Stonick, S. Titus, D. Weinlader, and S. Wolfer, "A multigigabit backplane transceiver core in $0.13 \mu \mathrm{m}$ CMOS with a power-efficient equalization architecture," IEEE J. Solid-State Circuits, vol. 40, no. 12, pp. 2658-2666, Dec. 2005.

[6] C. Menolfi, T.Toifl, P. Buchmann, M. Kossel, T. Morf, J. Weiss, and M. Schmatz, "A $16 \mathrm{~Gb} / \mathrm{s}$ source-series-terminated transmitter in $65 \mathrm{~nm}$ CMOS SOI," Dig. Tech. Papers, IEEE Int. SolidState Circuits Conf. pp. 446-447, 2007. 
[7] H. Hatamkhani, K.-L. J. Wong, R. Drost, C.-K. K. Yang, "A 10-mW 3.6-Gbps I/O transmitter," Dig. Tech. Papers, IEEE Symp. VLSI Circuits, pp. 97-98, 2003.

[8] J.-H. Song, K.-W. Kwon, J.-H. Chun, "A LowSwing Differential Voltage-Mode Driver with Preemphasis and Self-Diagnosis," Int. Tech. Conf. on Circuits/System, Computers and Communications, July 2010.

[9] K.-L. J. Wong, H. Hatamkhani, M. Mansuri, C.-K. K. Yang, "A 27-mW 3.6Gb/s I/O transceiver," IEEE J. Solid-State Ciucuits, vol. 39, no. 4, pp. 602-612, Apr. 2004.

[10] M. Kossel, C. Menolfi, J. Weiss, P. Buchmann, G. von Bueren, L. Rodoni, T. Morf, T. Toifl, and M. Schmatz, "A T-coil-enhanced $8.5 \mathrm{~Gb} / \mathrm{s}$ high-swing SST transmitter in $65 \mathrm{~nm}$ bulk CMOS with $<-16 \mathrm{~dB}$ return loss over $10 \mathrm{GHz}$ bandwidth," IEEE J. SolidState Circuits, vol. 43, no. 12, pp. 2905-2920, Dec. 2008.

[11] C. Yoo, K.-H. Kyung, K. Lim, H.-C. Lee, J.-W. Chai, N.-W. Choi, D.-J. Lee, and C.-H. Kim, "A 1.8-V 700-Mb/s/pin 512-Mb DDR-II SDRAM with on-die termination and off-chip driver calibration," IEEE J. Solid-State Circuits, vol. 39, no. 6, pp. 941-951, Jun. 2004.

[12] R. A. Philpott, J.S. Humble, R. A. Kertis, K. E. Fritz, B. K. Gilbert, and E. S. Daniel, "A 20Gb/s SerDes Transmitter with Adjustable Source Impedance and 4-tap Feed-Forward Equalization in 65nm Bulk CMOS," Proc. IEEE Custom Integrated Circuits Conference, pp. 623-626, 2008.

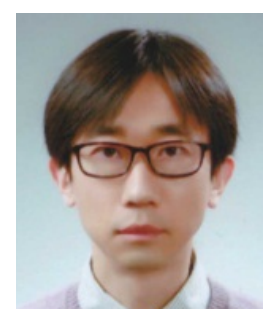

Chang-Hyun Bae received the B.S. degree in electronics engineering from Yeungnam University, Daegu, Korea, in 2001 and the M.S. degree in electronics engineering from Kyungpook National University, Daegu, Korea, in 2003. He joined

DRAM Design Team of Samsung Electronics, Hwasung, Korea, in 2003. He is also working toward the Ph.D. degree at Hanyang University. His research interests include high-speed interface circuit design.

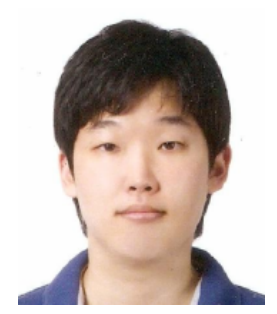

Dong-Ho Choi received the B.S. degree from Hanyang University, Seoul, Korea, in 2007, and is currently working toward the Ph.D. degree at Hanyang University. His research interests include clock and data recovery (CDR) circuit design.

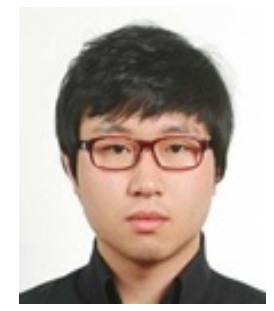

Keun-Seon Ahn received the B.S. degree in electrical and computer engineering from Hanyang University, Seoul, Korea, in 2010, and is currently working toward the Ph.D. degree at Hanyang University. His research interests include interface circuit design

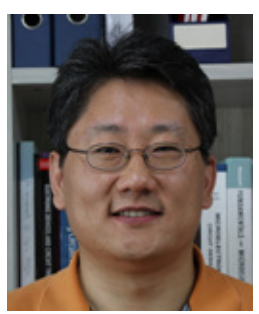

Changsik Yoo received the B.S. (with the highest honor), M.S., and Ph.D. degrees from Seoul National University, Seoul, Korea, in 1992 , 1994, and 1998, respectively, all in electronics engineering. From 1998 to 1999 , he was with Integrated Systems Laboratory (IIS), Swiss Federal Institute of Technology (ETH), Zurich, Switzerland, as a Member of Research Staff working on CMOS RF circuits. From 1999 to 2002, he was with Samsung Electronics, Hwasung, Korea. Since 2002, he has been a Professor of Hanyang University, Seoul, Korea. From 2008 to 2009, he was with Silicon Image Inc. Sunnyvale, California as a Senior Engineer to develop a HDMI receiver IC during the sabbatical leave. His main research interests include mixed-mode CMOS circuit, high-speed interface circuit, and power management integrated circuit. Dr. Yoo is the winner or co-winner of several technical awards including Samsung Best Paper Bronze Award in 2006 International SoC Design Conference, Silver Award in 2006 IDEC Chip Design Contest, Best Paper Award in 2006 Silicon RF IC Workshop, and Golden Prize for research achievement in the next-generation DRAM design from Samsung Electronics in 2002. He served as a member of technical committee of IEEE Int. Solid-State Circuits Conference (ISSCC) and is serving as a member of technical committee of VLSI Circuits Symposium (SOVC) and European Solid-State Circuits Conference (ESSCIRC). 